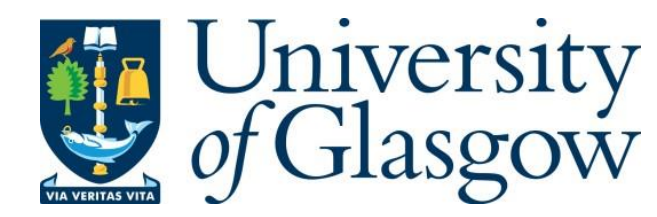

Kimonis, V. et al. (2020) NUBPL mitochondrial disease: new patients and review of the genetic and clinical spectrum. Journal of Medical Genetics, (doi: 10.1136/jmedgenet2020-106846).

There may be differences between this version and the published version. You are advised to consult the publisher's version if you wish to cite from it.

http://eprints.gla.ac.uk/217961/

Deposited on: 24 February 2021

Enlighten - Research publications by members of the University of Glasgow http://eprints.gla.ac.uk 


\section{NUBPL mitochondrial disease: new patients and review of the genetic and clinical spectrum}

Virginia Kimonis ${ }^{*, 1}$, Rehab al-Dubaisi ${ }^{1}$, Andrew E. Maclean ${ }^{2,3}$, Kathy Hall ${ }^{1}$, Lan Weiss ${ }^{1}$, Alexander E. Stover ${ }^{4}$, Philip H. Schwartz ${ }^{4}$, Bethany Berg ${ }^{1}$, Cheng Cheng ${ }^{1}$, Sumit Parikh $^{5}$, Blair R. Conner ${ }^{6}$, Sitao $\mathrm{Wu}^{6}$, Anton N. Hasso ${ }^{7}$, Daryl A. Scott ${ }^{8,9}$, Mary Kay Koenig ${ }^{10}$, Rachid Karam ${ }^{6}$, Sha Tang ${ }^{6}$, Moyra Smith ${ }^{1}$, Elizabeth Chao ${ }^{1,6}$, Janneke Balk², Eli Hatchwell ${ }^{11}$, Peggy S. Eis ${ }^{*, 12}$.

${ }^{1}$ Division of Genetics and Metabolism, Department of Pediatrics, University of California, Irvine, Children's Hospital of Orange County, Orange, CA, USA

${ }^{2}$ Department of Biological Chemistry, John Innes Centre, Colney Lane, Norwich, UK ${ }^{3}$ Current address: Wellcome Centre for Integrative Parasitology, University of Glasgow, 120 University Place Glasgow G12 8TA, UK

${ }^{4} \mathrm{CHOC}$ National Human Neural Stem Cell Resource, Children's Hospital of Orange County Research Institute, Orange, CA, USA

${ }^{5}$ Center for Pediatric Neurology, Cleveland Clinic, Cleveland, OH, USA

${ }^{6}$ Ambry Genetics, Aliso Viejo, CA, USA

${ }^{7}$ Radiological Sciences, School of Medicine, University of California, Irvine, CA, USA ${ }^{8}$ Department of Molecular and Human Genetics, Baylor College of Medicine, Houston, TX, USA

${ }^{9}$ Department of Molecular Physiology and Biophysics, Baylor College of Medicine, Houston, TX, USA

${ }^{10}$ Departments of Pediatrics and Neurology, The University of Texas Medical School at Houston, Houston, TX, USA

${ }^{11}$ Population Bio UK, Inc., Oxfordshire, UK

${ }^{12}$ Population Bio, Inc., New York, NY, USA 
Prepared for Journal of Medical Genetics, Original research manuscript

Word count: 4183

${ }^{*}$ Corresponding authors:

Virginia Kimonis, MD

Division of Genetics and Genomic Medicine

Department of Pediatrics

University of California, Irvine, Orange, CA 92868

Tel: (714) 456-5792, Fax: (714) 456- 5330,

Email address: vkimonis@uci.edu

Peggy S. Eis, PhD

Population Bio, Inc.

1120 Avenue of the Americas, Suite 1514

New York, NY 10036

Tel: (608) 692-9901

Email address: pegeis@populationbio.com 


\section{ABSTRACT}

Background The NUBPL gene was first reported as a cause of mitochondrial complex I deficiency (MIM 613621, 618242) in 2010. To date, only eight patients have been reported with this mitochondrial disorder. Five other patients were recently reported to have NUBPL disease but their clinical picture was different from the first eight patients. Here we report clinical and genetic findings in five additional patients (four families).

Methods Whole exome sequencing was used to identify patients with compound heterozygous NUBPL variants. Functional studies included RNA-Seq transcript analyses, missense variant biochemical analyses in a yeast model (Yarrowia lipolytica), and mitochondrial respiration experiments on patient fibroblasts.

Results The previously reported c.815-27T $>C$ branch-site mutation was found in all four families. In prior patients, c.166G>A [p.G56R] was always found in cis with c.815$27 \mathrm{~T}>\mathrm{C}$, but only two of four families had both variants. The second variant found in trans with c.815-27T >C in each family was: c.311T >C [p.L104P] in three patients, c. $693+1 \mathrm{G}>\mathrm{A}$ in one patient, and c.545T >C [p.V182A] in one patient. Complex I function in the yeast model was impacted by p.L104P but not p.V182A. Clinical features include onset of neurological symptoms at 3-18 months, global developmental delay, cerebellar dysfunction (including ataxia, dysarthria, nystagmus, and tremor), and spasticity. Brain magnetic resonance imaging showed cerebellar atrophy. Mitochondrial function studies on patient fibroblasts showed significantly reduced spare respiratory capacity.

Conclusion We report on five new patients with NUBPL disease, adding to the number and phenotypic variability of patients diagnosed worldwide, and review prior reported patients with pathogenic NUBPL variants. 


\section{INTRODUCTION}

Complex I deficiency is the third most common mitochondrial disorder and is highly heterogeneous, clinically and genetically. Human complex I has 44 different subunits that are encoded by nuclear and mitochondrial genes[1]. To date, pathogenic variants have been identified in 34 genes[2] and a molecular diagnosis is commonly achieved in $\sim 50 \%$ of affected individuals[3]. Additionally, there are at least 16 genes that play a role in the assembly of complex I, of which 12 have been identified to cause autosomal recessive disease[4,5]. One such assembly factor is nucleotide binding protein like (NUBPL), which was first identified as Ind1 in the yeast Yarrowia lipolytica[6]. Depletion of human or yeast NUBPL protein leads to decreased complex I activity[6, 7].

Variants in the NUBPL gene (MIM 613621) were first associated with complex I deficiency (MIM 618242) in 2010 in a whole exome sequencing (WES) study of over 100 patients with clinical and biochemical evidence of complex I deficiency[3]. One patient was identified initially with a point mutation in the paternal copy of NUBPL (c.166G>A [p.G56R]), while the maternal copy had a large chromosomal rearrangement that disrupts NUBPL. However, a protein variant carrying only the p.G56R substitution was able to rescue complex I activity in patient fibroblasts, suggesting it was not pathogenic[8]. Further sequence analysis revealed an intronic variant, c.815-27T $>C$ on the paternal copy, which affects a splicing branch site[3, 8]. A subset of the c.815-27T>C transcripts lack exon 10, leading to a frameshift and truncated protein product. Interestingly, the c.815-27T>C variant is found in $\sim 1 \%$ of European ancestry subjects (Finnish plus non-Finnish) in the Genome Aggregation Database (gnomAD)[9].

Subsequently, six additional patients were identified from a magnetic resonance imaging (MRI) database of more than 3,000 subjects with unclassified leukoencephalopathy. These cases were recognized by specific MRI features including diffuse leukodystrophy involving the cerebellar cortex, periventricular deep and subcortical white matter and corpus callosum with some cystic changes[10]. All patients from this study had c.815-27T >C in cis with c.166G $>$ A plus a second deleterious NUBPL variant in trans, with the exception of one patient that is presumed to be homozygous for c.815-27T>C and c.166G>A. With recent reports on three more 
families, there are now 14 patients (in 11 families) reported to have NUBPL disease[3, 10-15]. Here, we now report clinical features of five additional patients in four families, plus functional and biochemical experiments as supporting evidence for complex I deficiency in these patients. We also review the spectrum of genotypes and phenotypes found in all known cases of NUBPL disease.

\section{MATERIALS AND METHODS}

\section{Patients}

Institutional Review Board (IRB) approval was obtained from the University of California, Irvine for this study. Informed consents were obtained from the parents of the children who participated in this study, in addition to consent from one patient over the age of 18 years.

WES was used for identification of the NUBPL variants at Baylor Genetics (Houston, TX), at GeneDx (Gaithersburg, MD), or as described previously[12, 16, 17], with the exception of Patient 1B (Sanger sequencing). Thoughout the manuscript, all NUBPL variants are described based on NM_025152.3. Brain MRIs for all patients and a cerebellar brain biopsy for Patient $1 \mathrm{~A}$ were provided by the referring physicians.

\section{RNA splicing analysis}

Splicing analysis of NUBPL variant c.815-27T>C was performed using CloneSeq, an RNA sequencing (RNA-Seq) assay based on cloning of RT-PCR products followed by massively parallel sequencing of the cloned transcripts[18]. Briefly, total RNA was isolated from whole blood from the proband, both parents, an affected sibling, and an unaffected sibling of Family 1. RT-PCR was perfomed on cDNA derived from mRNA only using the following primers: 5'-ATGGTATTGCTTGTATGTCTATGG-3', and 5'GTTCCATCACCACATTGCTG-3'. Transcript levels (Sashimi plots) and the percentage of aberrantly spliced transcripts was determined as described previously[18].

\section{Mitochondrial respiration assays}


The Seahorse assay system (Agilent, Santa Clara, CA), which directly measures the oxygen consumption rate (OCR), was used for mitochondrial respiration studies[19]. Human fibroblasts from three NUBPL patient cell lines (patients $1 \mathrm{~A}, 1 \mathrm{~B}$, and 2) and one control line were cultured in DMEM supplemented with $10 \%$ fetal bovine serum, $5 \mathrm{mM}$ Glucose, $1 \mathrm{mM}$ pyruvate, and $1 \mathrm{X}$ non-essential amino acids, penicillin, streptomycin, and amphotericin B (Gibco). The day prior to the assay, patient and control fibroblasts were seeded onto 24-well Seahorse XF24 Cell Culture Microplates at a density of 30,000 cells $/$ well $\left(\sim 1 \times 10^{5}\right.$ cells $\left./ \mathrm{cm}^{2}\right)$ and incubated overnight in a humidified $5 \% \mathrm{CO}_{2}$ $37^{\circ} \mathrm{C}$ incubator.

After 18 hours incubation, cells were assayed using a Seahorse XF Cell Mito Stress Test Kit and XFe24 analyzer according to the manufacturer's protocol[19]. Cellular bioenergetic profiles were measured by three serial injections of four reagents at $1 \mu \mathrm{M}$ final concentration each: oligomycin, which inhibits ATP synthase (complex V); carbonyl cyanide-4 (trifluoromethoxy) phenylhydrazone (FCCP), a proton ionophore that induces uncoupling of ATP synthesis from electron transfer and is thus a measure of maximal respiration; and a mixture of rotenone plus antimycin $A$, which completely inhibits electron transport to enable measurement of non-mitochondrial respiration. Data were normalized to total protein per well using the Bio-Rad DC protein assay. The XF Mito Stress Test report generator was used to calculate basal respiration, ATP turnover rate, proton leak, and maximal and spare respiratory capacity for each cell line (patient's fibroblasts were assayed in triplicate).

\section{Yeast model functional studies}

The functional effect of the NUBPL variants was tested in Yarrowia lipolytica as previously described[12, 20].

\section{RESULTS}

We report on genetic and clinical findings in four families, three of which contain individuals with mitochondrial dysfunction symptoms consistent with complex I deficiency due to autosomal recessive NUBPL mutations. Pedigree charts for the 
families are shown in Figure 1A (Family 1 has two patients) and brain MRIs for the patients are shown in Figure 1B. Cerebellar brain biopsy results are shown in Figure 1C for Patient 1A. Detailed clinical descriptions for each patient are reported in the Supplementary Data file. Below, molecular, clinical, and functional findings are reported for the five new patients plus genotype-phenotype comparisons to all previously reported NUBPL patients.

\section{Molecular diagnosis of new patients}

Genetic analysis using WES revealed compound heterozygous variants in the NUBPL gene (Tables 1 and 2). All patients were heterozygous for the common c.815-27 T>C variant (present in $\sim 1 \%$ of European ancestry subjects) found in the first reported case[3]. The missense variant c.166G $>A$ [p.G56R], present in cis with c.815-27T $>C$ in all previously reported cases, was found in Families 2 and 3 (Figure1A, Table 1). However, we report, for the first time, patients without c.166G>A, see Families 1 and 4 (Figure 1A, Table 1).

Missense variant c.311T>C [p.L104P] was found in trans with c.815-27T>C in Families 1 and 3 . It is predicted to be damaging/deleterious (Table 2) and was shown to be pathogenic in a yeast model[12]. It was first reported in a 2014 WES study[16] to cause complex I deficiency in Patient 1A; however, no clinical details were provided. Subsequently, it was reported for two conditions[11, 13] with overlapping phenotypes (see below) wherein patients are compound heterozygotes with missense variants p.D96V or p.F242L (Tables 1, 2) instead of c.815-27T>C.

In Family 2, the patient was found to be compound heterozygous for previously reported variants[10], c.815-27T>C and c.166G $>A$ in cis plus the splice donor mutation c.693+1G>A in trans (Figure 1A, Table 1). In Family 4, the patient was found to be compound heterozygous for $\mathrm{C} .815-27 \mathrm{~T}>\mathrm{C}$ and newly reported variant c.545T $>\mathrm{C}$ [p.V182A], which is predicted to be damaging/deleterious (Table 2) but our yeast model in vitro functional assays (see below) do not support its pathogenicity. 
Table 1. Clinical summary for 5 new patients and previous 14 patients reported with autosomal recessive NUBPL disease.

\begin{tabular}{|c|c|c|c|c|c|c|c|c|c|c|c|c|}
\hline $\begin{array}{l}\text { Family } \\
\text { Country }\end{array}$ & Patient & $\begin{array}{l}\mathrm{Age}^{\mathrm{a}} \\
(\mathrm{yr})\end{array}$ & $\begin{array}{l}\text { Age of Gender } \\
\text { onset } \\
\text { (mo) }\end{array}$ & NUBPL variants & Inh. & $\begin{array}{l}\text { Dev. } \\
\text { delay }\end{array}$ & $\begin{array}{l}\text { Clinical } \\
\text { features }^{c}\end{array}$ & $\begin{array}{l}\text { Cerebellar } \\
\text { atrophy } \\
\text { (MRI) }^{d}\end{array}$ & $\begin{array}{l}\text { Cl def. } \\
\text { defect }^{\mathrm{e}}\end{array}$ & $\begin{array}{l}\text { High } \\
\text { lactate }^{f}\end{array}$ & $\begin{array}{l}\text { Other } \\
\text { clinical } \\
\text { details }^{\mathrm{g}}\end{array}$ & Ref \\
\hline
\end{tabular}

\section{New patients - compound heterozygotes with c.815-27T>C}

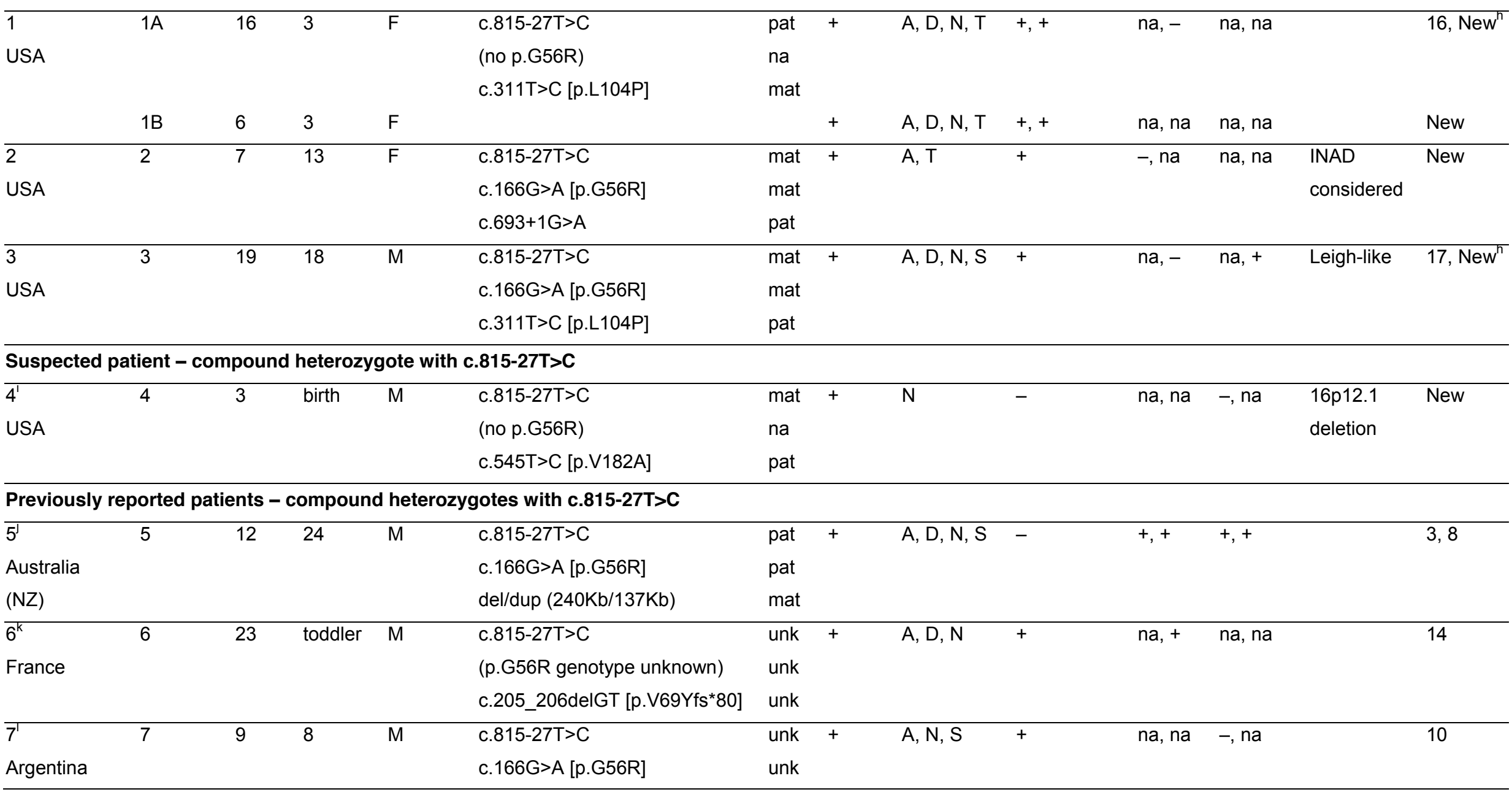




\begin{tabular}{|c|c|c|c|c|c|c|c|c|c|c|c|c|c|}
\hline & & & & & (hemi. or hom. suspected) & & & & & & & & \\
\hline 8 & 8 & 12 & 8 & $\mathrm{M}$ & c. $815-27 T>C$ & & + & $A, D, N, S$ &,-+ &,+ na &,++ & & 10 \\
\hline \multirow[t]{2}{*}{ Germany } & & & & & c.166G>A [p.G56R] & pat & & & & & & & \\
\hline & & & & & c.667_668ins ${ }^{m}{ }^{\text {[p.E223Afs*4] }}$ & mat & & & & & & & \\
\hline$\overline{9}$ & $9 \mathrm{~A}$ & 9 & 13 & $\mathrm{~F}$ & c.815-27T>C & pat & + & $A, D, N$ & + &,+- &,- na & & 10 \\
\hline \multirow[t]{3}{*}{ Canada } & & & & & c.166G>A [p.G56R] & pat & & & & & & & \\
\hline & & & & & c.313G>T [p.D105Y] & mat & & & & & & & \\
\hline & $9 \mathrm{~B}$ & 7 & 13 & $\mathrm{~F}$ & & & + & $A, D, N$ &,-+ &,++ &,++ & & \\
\hline$\overline{10}$ & 10 & 7 & 21 & $\mathrm{~F}$ & c. $815-27 \mathrm{~T}>\mathrm{C}$ & pat & + & $A, D, N, S$ & - & na, na &,+ na & & 10 \\
\hline \multirow[t]{2}{*}{ USA } & & & & & c. $166 G>A$ [p.G56R] & pat & & & & & & & \\
\hline & & & & & c. $693+1 G>A$ & unk & & & & & & & \\
\hline$\overline{11}$ & 11 & 4 & 10 & $\mathrm{~F}$ & c.815-27T>C & unk & + & $A, D, S$ & - &,++ &,++ & & 10 \\
\hline \multirow[t]{2}{*}{ Netherlands } & & & & & c. $166 G>A$ [p.G56R] & unk & & & & & & & \\
\hline & & & & & c.579A>C [p.L193F] & unk & & & & & & & \\
\hline$\overline{12}$ & 12 & na & na & na & c.815-27T>C & unk & na & na & na & na, na & na, na & & 12 \\
\hline \multirow[t]{2}{*}{ Germany } & & & & & (p.G56R genotype unknown) & unk & & & & & & & \\
\hline & & & & & c.859G $>$ T [p.G287C] & unk & & & & & & & \\
\hline \multicolumn{14}{|c|}{ Previously reported patients - compound heterozygotes without c.815-27T>C } \\
\hline 13 & $13 \mathrm{~A}$ & 25 & toddler & $\mathrm{F}$ & c.311T>C [p.L104P] & mat & + & $\mathrm{D}, \mathrm{H}$ & + & na, na & na, na & dystonia, & 11 \\
\hline \multirow[t]{3}{*}{ UK } & & & & & c.287A > T [p.D96V] & pat & & & & & & BSN & \\
\hline & $13 \mathrm{~B}$ & 17 & toddler & $\mathrm{F}$ & & & + & $\mathrm{A}, \mathrm{T}$ & + & na, na & na, na & dystonia, & \\
\hline & & & & & & & & & & & & BSN & \\
\hline$\overline{14}$ & 14 & 13 & 18 & $\mathrm{~F}$ & c.311T>C [p.L104P] & mat & + & $\mathrm{H}$ &,-- & na, + &,+ na & multi- & 13 \\
\hline UK & & & & & c.726C>G [p.F242L] & pat & & & & & & systemic & \\
\hline$\overline{15^{n}}$ & $15 \mathrm{~A}$ & 2 & 4 & $\mathrm{M}$ & c. $693+1 \mathrm{G}>\mathrm{A}$ & pat & + & $\mathrm{H}, \mathrm{N}, \mathrm{S}$ &,++ &,+ na &,+ na & thalamic & 15 \\
\hline \multirow[t]{3}{*}{ USA } & & & & & c.351G>A [p.M117I] & mat & & & & & & involvement & \\
\hline & $15 B$ & 2 & 4 & M & & & + & $\mathrm{H}, \mathrm{N}, \mathrm{S}$ &,++ & + , na &,+ na & thalamic & \\
\hline & & & & & & & & & & & & involvement & \\
\hline
\end{tabular}

${ }^{a}$ Age at last clinical assessment (rounded to whole year) 


\footnotetext{
${ }^{\mathrm{b}}$ Inheritance (Inh.) indicated as paternal (pat), maternal (mat), unknown (unk); not applicable (na) listed for patients that did not have the c.166G>A [p.G56R] variant ${ }^{\mathrm{c}}$ Common clinical features: $\mathrm{A}=$ ataxia, $\mathrm{D}=$ dysarthria, $\mathrm{H}=$ hypotonia, $\mathrm{N}=$ nystagmus, $\mathrm{S}=$ spasticity, $\mathrm{T}=$ tremor (intention)

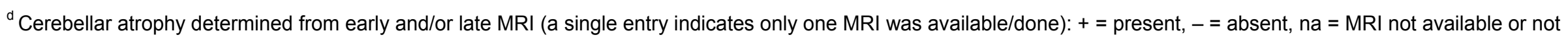
done

${ }^{\text {e }}$ Complex I (Cl) deficiency (def.) defect reported for fibroblast (first entry) or muscle (second entry): + = present, $-=$ absent, na $=$ test data not available or not done ${ }^{\dagger}$ High lactate reported for plasma (first entry) or CSF (second entry): + = present, $-=$ absent, na = test data not available or not done

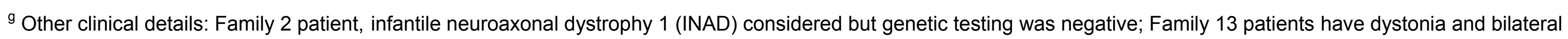
striatal necrosis (BSN); Family 14 patient is reported to have multi-systemic involvement, including renal tubular acidosis, osteoporosis, hepatomegaly, growth hormone deficiency (short stature present), and interstitial lung disease

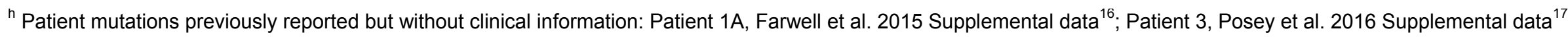

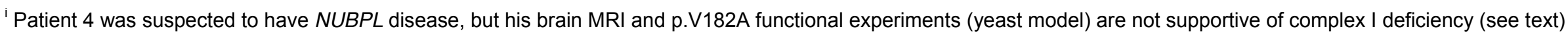
${ }^{\prime}$ Family 5 was reported to be from Australia by Kevelam et al. $2013^{10}$, but is actually from New Zealand (NZ) per personal communication from D. Thorburn

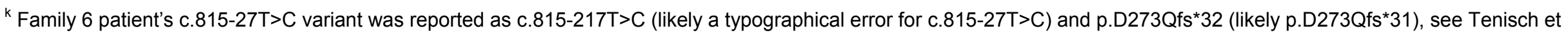
al. $2012^{14}$

' Family 7 patient is thought to be hemizygous or homozygous for c.166G>A [p.G56R] and c.815-27T>C [p.D273Qfs*31], see Discussion

${ }^{\mathrm{m}}$ Full variant name is c.667_668insCCTTGTGCTG

${ }^{\mathrm{n}}$ Patients $15 \mathrm{~A}$ and $15 \mathrm{~B}$ are monozygotic twins
} 
Table 2. Variants reported in autosomal recessive NUBPL disease ${ }^{a}$ : functional impact and population frequency.

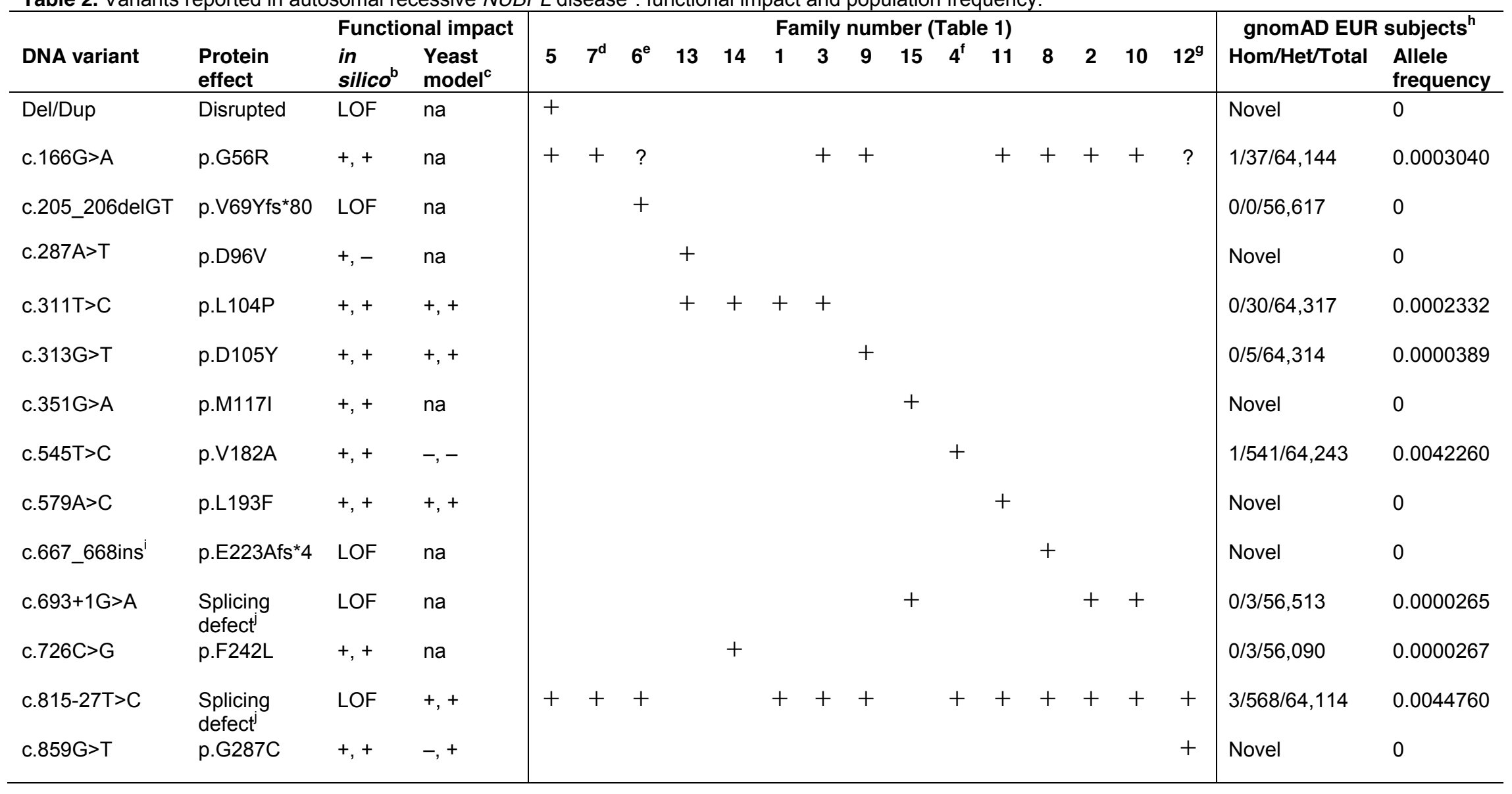




\footnotetext{
${ }^{a}$ See Table 1 Reference column (Ref) for patient case report citations.

${ }^{\mathrm{b}}$ In silico prediction (LOF or Polyphen2, SIFT): loss-of-function (LOF); Polyphen2, + = possibly or probably damaging, $-=$ = benign; SIFT, + = deleterious, $-=$ tolerated

${ }^{\mathrm{c}}$ Yeast model (Yarrowia lipolytica) study, see Maclean et al. $2018^{12}$ and Figure 4: first entry, $+=$ severely or slightly decreased complex I level, $-=$ normal complex I level; second entry, + = impaired growth in cold, - = normal growth in cold; na = not applicable or not done

${ }^{d}$ Family 7 patient is thought to be hemizygous or homozygous for c.166G>A [p.G56R] and c.815-27T>C [p.D273Qfs*31], see Discussion

${ }^{\text {e Family }} 6$ patient's c.166G>A [p.G56R] genotype is unknown (?); his c.815-27T>C variant was reported as c.815-217T>C (likely a typographical error for c.81527T>C) and p.D273Qfs*32 (likely p.D273Qfs*31), see Tenisch et al. $2012^{14}$

${ }^{f}$ Patient 4 was suspected to have NUBPL disease, but his brain MRI and p.V182A functional experiments (yeast model) are not supportive of complex I deficiency (see text)

${ }^{g}$ Family 12 patient's c.166G>A [p.G56R] genotype is unknown (?)

${ }^{\mathrm{h}}$ gnomAD is the Genome Aggregation Database, see Karczewski et al. 2019 ${ }^{9}$ : EUR subjects correspond to Non-Finnish European (NFE) ancestry; population information reports the number of homozygous (Hom), heterozygous (Het), and total (Total) subjects with the variant and the corresponding allele frequency 'Full variant name is c.667_668insCCTTGTGCTG

${ }^{j}$ Splicing defects are: c.693+1G $>$ A impacts a splice donor site; c.815-27T $>C$ is a branch migration variant that causes skipping of exon 10 and a frameshift (p.D273Qfs*31)
} 


\section{Clinical findings of new patients}

Primary clinical features of Patients 1-5 (3 females and 2 males) are summarized in Table 1; detailed clinical descriptions for each patient are provided in the Supplementary Data file. All patients are of European ancestry (USA-based) and at last evaluation are aged 3-19 years. Onset of neurological symptoms ranged from birth to 18 months and included global developmental delay, cerebellar dysfunction (ataxia, dysarthria, dysmetria, nystagmus, and intentional tremor), hyperreflexia with clonus, and, in Patient 3, a Leigh-like phenotype. Patient 2 was initially thought to have infantile neuroaxonal dystrophy 1 (INAD, MIM 256600) and Patient 4 also has a 16p12.1 deletion[21], which may be contributing to his symptoms.

All five patients had delays in their gross motor skills and are able to walk with assistance. Gaits of Patients $1 \mathrm{~A}$ and 2 were described as wide-based and ataxic, while Patient 3 walked with a narrow/scissoring (diplegic) gait. All patients have difficulties with their fine motor skills (especially with tremor) except Patient 2, whose tremor has improved with the help of occupational therapy and, potentially (parental report, see Supplementary data), from treatment with EPI-743. Patients $1 \mathrm{~A}$ and 4 have language delay while the others have slurred speech. Patient 3 has right sensorineural hearing loss. Cognition level varies, but is impaired for all except for Patient 2 who has a normal IQ. Brain MRIs for all five patients are shown in Figure 1B. Cerebellar atrophy was noted in all patients except for Patient 4. Cerebellar biopsy results for Patient $1 \mathrm{~A}$ are shown in Figure 1C.

\section{Functional studies of new patients}

Aberrant splicing is observed for NUBPL c.815-27T >C carriers

Transcript variants in Family 1 carriers and non-carriers of NUBPL c.815-27T>C were assessed by CloneSeq analysis[18]. The two most abundant abnormal transcripts were r.815-72_815-1ins72 (partial intron 9 inclusion) and r.815_897del83 (exon 10 skipping), as shown in the Sashimi plots (Figure 2A). Transcript variant r.815-72_815-1ins72 was 
essentially not observed in non-carriers of c.815-27T $>C$, but was found in the carriers (observed reads were 239 for Sister+, 198 for Proband+, and 129 for Father+).

Whereas transcript variant r.815_897del83 was observed in all members of Family 1, but higher levels were found in carriers of c.815-27T>C (observed reads were 629 for Sister+, 410 for Proband+, and 801 for Father+) compared to non-carriers (observed reads were 245 for Mother- and 59 for Sister-). Relative levels of the two transcript variants, r.815-72_815-1ins72 and r.815_897del83, are reported as PSI (Figure 2B), a measure of how efficiently sequences of interest are spliced into transcripts. This analysis shows that $5-20 \%$ of transcripts expressed by $\mathrm{c} .815-27 \mathrm{~T}>\mathrm{C}$ carriers contain partial intron 9 inclusion (r.815-72_815-1 ins72) but it is not observed in non-carriers. Skipping of exon 10 (r.815_897del83) was observed in 25\% of transcripts expressed by the carriers, but only $\sim 5 \%$ of non-carriers. These results are consistent with aberrant splicing data previously reported for a complex I deficiency patient with the c.815-27T >C variant[8].

Mitochondrial function is impaired in patient fibroblasts

Fibroblasts from three NUBPL patient cell lines (Patients 1A, 1B, and 2) and one control cell line were used to perform in vitro mitochondrial respiration studies (Figure 3). Basal respiration was not significantly different in the NUBPL patient fibroblast cells compared to control cells, nor was ATP production (post-oligomycin injection) or non-mitochondrial respiration (post-rotenone + antimycin A injection). However, spare respiratory capacity, which is the difference between maximal respiration (post-FCCP injection) and basal respiration, was significantly reduced in all three NUBPL patient fibroblast cells as compared to the control cells (Figure $3 \mathrm{~B}$ ). This is in contrast to the negative results found by the clinical electron transport chain analyses of Patient 1A's muscle or Patient 2's fibroblasts (see Table 1).

Functional studies of NUBPL variants in the yeast model Yarrowia lipolytica 
The c.815-27T>C variant, found in all four families, was previously shown to result in synthesis of a truncated protein, p.D273QfsX31, in addition to reduced amounts of normal NUBPL protein[8]. The equivalent truncated protein product in Yarrowia, p.N271QfsX31, is less stable and severely affected the assembly of complex I[20].

The p.L104P substitution, present in Families 1 and 3, changes a highly conserved amino acid in a protein motif involved in ATP hydrolysis. The equivalent substitution caused instability of the Yarrowia Ind1 protein and decreased complex I levels to $\sim 30 \%$ of the control line[12].

The p.V182A substitution is only found in Family 4. In the Yarrowia Ind1 protein, valine is semi-conserved and aligns with a methionine (Figure 4A). Therefore, to match the human sequence, first methionine was substituted by valine (p.M180V), both of which have large non-polar side chains. Next, methionine was changed to alanine (p.M180A), which has a much shorter side chain. These changes were neutral with respect to complex I levels (Figure 4B).

When the Yarrowia IND1 gene is deleted (ind1 $\Delta$ ), a 160-kDa assembly intermediate containing the NUCM subunit (NDUFS2 in human) accumulates[12]. This intermediate has been observed in all Ind1 protein variants tested so far, including the p.G136D variant (p.G138D in human), which has otherwise no discernable phenotypes. In contrast, the assembly intermediate was not observed in p.M180V or p.M180A (Figure 4C). Another feature of ind1 mutants is a striking growth defect at low temperature[12]. When the p.M180V and p.M180A variants were grown at $10 \mathrm{C}$, colonies grew to the same size as in the control strain, whereas the ind $1 \Delta$ mutant did not grow at all (Figure 4D). Taken together, the biochemical and phenotypic tests in Yarrowia yeast show that substitution of M180 with valine or alanine in Ind1 does not have an effect on complex I, but they do not rule out a potential effect of the valine to alanine substitution on the function of human NUBPL. We note that the V182A variant is predicted to be possibly damaging/deleterious (Table 2).

\section{Clinical comparison of all NUBPL patients}


Table 1 summarizes the main clinical features in our five new patients reported here, plus twelve previously reported patients (19 patients total in 15 families). Patients are sub-grouped based on whether they have the c.815-27T>C branch-site mutation, which is found in 14 of 19 patients. Phenotypes for this subset of patients have greater overlap than with patients without the c.815-27T>C variant (Families 13-15). We note that clinical information was not available for German Patient 12 and USA Patient 4 is listed separately as NUBPL disease is suspected but not supported by his brain MRI (Figure 1B) or functional experiments on the p.V182A variant (Figure 4).

Notably, all patients had developmental delay with onset as infants or toddlers. Cerebellar dysfunction symptoms (ataxia, dysarthria, nystagmus, and tremor) were prevalent in patients with the c.815-27T $>C$ branch-site mutation, but absent (Patient 14) or minimal (Patients 13A,13B, 15A, and 15B) in patients without this mutation. Spasticity was reported in 6 of 14 patients with c.815-27T >C and 3 of 5 patients without this mutation. Brain MRIs revealed cerebellar atrophy in 13 of 18 patients who underwent at least one assessment but this symptom may not be present at an early age (e.g., Patients 8 and 9B were only positive in a later MRI and Patients 5, 10 and 11 were negative in an early MRI but no late MRI results were available).

Biochemical assays for complex I defects (electron transfer) and lactate levels (plasma and/or CSF) can be inconclusive. For example, results were reported for 12 of 19 patients but only 9 of 12 patients tested positive for a complex I defect and/or a high lactate level.

Patient 2 is the first unrelated case of NUBPL disease that has identical mutations to those reported in a previous case (Patient 10, Table 1)[10]. Ataxia is the main overlapping cerebellar symptom for these two patients and both were noted to have normal intelligence. Cerebellar atrophy was noted in Patient 2's MRI obtained at age 3 years (Figure 1B), but not in Patient 10's MRI obtained at age 1.5 years old (no late MRI was available). Previously reported Patients $15 \mathrm{~A}$ and $15 \mathrm{~B}[15]$ also have c. $693+1 \mathrm{G}>\mathrm{A}$ (but lack c.815-27T $>\mathrm{C}$ ) and have thalamic involvement in addition to cerebellar atrophy.

Patients $1 \mathrm{~A}, 1 \mathrm{~B}$, and 3 share nearly identical variants (patients $1 \mathrm{~A}$ and $1 \mathrm{~B}$ do not have c.166G>A [p.G56R]) and their overlapping cerebellar symptoms include ataxia, 
dysarthria, and nystagmus, while spasticity was only reported in Patient 3. All three patients showed cerebellar atrophy in their MRIs (Figure 1B) and electron transport chain assays for Patients $1 \mathrm{~A}$ and 3 were both negative for a complex I defect (Table 1). Interestingly, while previously reported Patients $13 \mathrm{~A}, 13 \mathrm{~B}$, and $14[11,13]$ share the c.311T>C [p.L104P] mutation (but lack c.815-27T>C) with Patients 1A, 1B, and 3, their main clinical features are distinct (Table 1). Patients 13A and 13B have dystonia and bilateral striatal necrosis (BSN) and Patient 14 lacks cerebellar symptoms but has renal tubular acidosis, osteoporosis, hepatomegaly, and growth hormone deficiency.

\section{Genetic comparison of all NUBPL patients}

Table 2 summarizes the genetic findings for our 5 new patients plus 14 prior reported patients (19 patients total in 15 families). As noted above, only two patients (Patients 2 and 10) are compound heterozygous for identical mutations (c.166G $>A$ in cis with c.815-27T >C plus c.693+1G>A in trans).

Nearly all families (12 of 15 ) carry the c.815-27T >C branch-site mutation, which is not surprising since they are of European descent and this is the highest frequency NUBPL variant amongst known pathogenic variants (Table 2), based on the gnomAD database ( $0.45 \%$ in non-Finnish Europeans)[9]. Despite its relatively high frequency in the general population, aberrant splicing data, demonstrated in Patient 5[8] and in newly reported Patients $1 \mathrm{~A}$ and $1 \mathrm{~B}$ and their father (Figure 2), supports its pathogenicity.

While the $\mathrm{c} .815-27 \mathrm{~T}>\mathrm{C}$ branch-site mutation was reported to be hemizygous or homozygous in Patient 7[10], parental DNA was unavailable for testing so it is possible the patient is heterozygous for $\mathrm{c} .815-27 \mathrm{~T}>\mathrm{C}$ and has another yet to be characterized pathogenic NUBPL variant. We note that the gnomAD database reports ten c.815-27T> homozygotes (three non-Finnish European and seven Finnish subjects). It is unlikely that subjects with early-onset NUBPL disease would be included in a population database. We hypothesize that homozygous subjects have a mild form of NUBPL disease (e.g., many may be undiagnosed) or, as Tucker et al.[8] suggested, may be at higher risk of developing late-onset neurological disorders such as Parkinson's disease. 
As noted above, we report, for the first time, three patients without the c.166G>A [p.G56R] variant in cis with c.815-27T>C. The allele frequency difference between the c. $166 \mathrm{G}>\mathrm{A}(0.03 \%)$ and $\mathrm{c} .815-27 \mathrm{~T}>\mathrm{C}(0.45 \%)$ variants infers that they are not always found on the same haplotype. Despite in silico prediction that c.166G>A [p.G56R] is damaging/deleterious (Table 2), in vitro functional assays do not, thus far, support pathogenicity[8].

Besides c.815-27T >C and c.693+1G >A, c.311T>C [p.L104P] (allele frequency $0.02 \%$ ) is the only other pathogenic variant found in two or more families (see Families $1,3,13$, and 14). As noted above, for patients that share the c.311T >C [p.L104P] variant, their clinical symptoms are quite variable depending on which of the three pathogenic NUBPL variants (c.815-27T>C, c.287A>T [p.D96V], or c.726C>G [p.F242L]) they carry in trans (Tables 1, 2). Finally, we note that 6 of 15 families $(5,8,11,12,13$, and 15) carry a novel NUBPL variant (i.e., not reported in the gnomAD) in trans with a variant found in the general population.

\section{DISCUSSION}

Primary mitochondrial diseases (PMD) are caused by mutations in a large number of mitochondrial and nuclear genes, resulting in a broad range of phenotypes that are often present in other diseases[22]. As a subclass of PMD, complex I deficiency is one of the most common, with 34 nuclear genes now recognized to cause autosomal recessive disease[2]. Complex I assembly factor gene NUBPL (MIM 613621), 1 of 12 assembly factors known to cause disease[2,5] was first reported to cause complex I deficiency (MIM 618242) in 2010[3]. Prior to this report on clinical and genetic findings in 5 new patients, only 14 cases were known worldwide[3, 10-15].

We have presented evidence that four of our five patients have NUBPL complex I deficiency symptoms consistent with other known patients (all patients are summarized in Table 1). In addition to cerebellar atrophy (Table 1), brain MRIs (Figure 1B) showed progressive global cerebellar hypoplasia with both vermis and cortex involved, in addition to hypoplasia of the medulla and pons. Consistent with cerebellar involvement, ataxia, dysarthria, nystagmus, and tremor were usually found, which was also noted in 
prior patients, along with spasticity. Biochemical findings (e.g., complex I defects and high lactate), when available, were not a consistent indicator of mitochondrial dysfunction in our patients or prior patients. Patient 4 was suspected of having NUBPL disease, but neither his brain MRI (Figure 1B) or yeast model functional experiments for his c.545T>C [p.V182A] variant (Figure 4) are supporting evidence. He also has a 16p12.1 deletion (Figure 1A, Table 1) and this possibly explains his neurological symptoms[21].

None of the four NUBPL variants found in our five new patients (1A, 1B, 2, and 3) with NUBPL disease are novel. Variants c.815-27T>C and c.166G>A [p.G56R], reported to be in cis for most patients[3,10], were found in Patients 2 and 3 . However, Patients 1A and 1B (and suspected Patient 4) are the first reported patients that do not have c. $166 \mathrm{G}>\mathrm{A}$. This supports the conclusion by Tucker et al.[8] that c.815-27T>C, by itself, is a pathogenic variant as their in vitro experiments did not show that p.G56R was deleterious. Our Patient 2 is the first reported case with identical mutations (c.815$27 \mathrm{~T}>\mathrm{C}$ and c.166G $>\mathrm{A}$ in cis plus c.693+1G $>\mathrm{A}$ in trans) as a prior patient[10]. Variant c.311T>C [p.L104P], found in Families 1 and 3 (Figure 1A), was originally reported for Patient $1 \mathrm{~A}$ without a clinical description[16]. Recently it was reported in compound heterozygous patients that had either c.287A>T [p.D96V][11] or c.726C>G [p.F242L][13], but their phenotypes (see Table 1, Patients 13A, 13B, and 14) were distinct from our patients and prior patients that all had in common the c.815-27T $>C$ variant. Similarly, Patients $15 \mathrm{~A}$ and $15 \mathrm{~B}$, who also lack c.815-27T $>C$, have distinctive clinical features (e.g., thalamic involvement)[15]. They have the same splicing mutation (c.693+1G>A) as Patients 2 and 10 plus a novel mutation (p.M117I). These findings underscore that, even for a single gene, phenotypes can vary significantly.

On a broader level, there are two observations in common between NUBPL and three other complex I assembly factors: NDUFAF5, NDUFAF6, and NDUFAF8. First, NDUFAF6 has also been linked to dystonia and BSN[23], which are clinical findings in NUBPL patients that are compound heterozygous for p.D96V and p.L104P[11]. Second, analgous to NUBPL c.815-27T>C, aberrant splicing variants that do not involve a splice donor or acceptor have been reported for NDUFAF5 (c.223-907A>C)[24] and NDUFAF8 (c.195+271C>T)[25]. Such variants may be difficult to uncover in WES bioinformatics 
pipelines that exclude intronic and/or common variants. This is a particularly important issue for $\mathrm{c} .815-27 \mathrm{~T}>\mathrm{C}$ due to its $\sim 1 \%$ frequency in the European ancestry population (i.e., we suspect that complex I deficiency patients with this splicing mutation are underdiagnosed).

With such a limited number of NUBPL disease patients, now eighteen total with our four new patients, there are several open genotype-phenotype questions. First, with additional evidence that $\mathrm{c} .815-27 \mathrm{~T}>\mathrm{C}$ by itself is pathogenic (Patients $1 \mathrm{~A}$ and $1 \mathrm{~B}$ ), what phenotypes, if any, are found in c.815-27T >C homozygotes (ten are reported in gnomAD)? Second, does c.815-27T>C confer an alternate clinical picture compared to patients without it? Third, despite lack of evidence thus far from in vitro studies, does p.G56R contribute to the complex I deficiency phenotype? Fourth, what genetic backgrounds and/or environmental factors contribute to varying degrees of severity in patients with the same mutations (either siblings or in unrelated patients like Patients 2 and 10 in Table 1)? Finally, new treatments are needed, which are currently limited to mitochondrial cocktails (e.g., supplements of coenzyme Q10, carnitine, and a-lipoic acid) although experimental drugs have shown promise (e.g., EPI-743)[26, 27]. 


\section{Acknowledgements}

We thank the families for their permission to present their medical history and all the health care providers involved in the management of the patients. We also thank the families involved with raising the awareness for patients with NUBPL disease via the NUBPL Foundation (nubpl.org) and the Spooner Girls Foundation for funding. We thank Dr. Klemens Hertel, Department of Microbiology Molecular Genetics, UC Irvine, Irvine, CA for his technical expertise on RNA splicing. Special acknowledgement is given to Dr. Mari Perez-Rosendahl, Department of Pathology, University of California Irvine, Orange, $\mathrm{CA}$, for the photomicrographs of the brain biopsy sample from Patient $1 \mathrm{~A}$.

\section{Contributors}

VK and PSE established the study and recruited patients. VK, SP, DAS, and MKK provided patient/family samples and clinical information. LW, KH, ST, MS, and EC performed and analyzed the genetic experiments. AEM, AES, PHS, BB, BRC, SW, RK, and JB performed and/or analyzed the functional and/or biochemical experiments. ANH provided analyses of MRIs. VK, RAD, KH, MS, EC, EH, and PSE analyzed and interpreted clinical and/or genetic data. VK, RAD, and PSE wrote the manuscript; CC, $\mathrm{JB}$, and $\mathrm{EH}$ provided critical revisions.

\section{Funding}

Funding for these studies were provided by the Spooner Girls Foundation, CART (Center for Autism Research and Translation), and the ICTS (Institute of Clinical Translational Science, UC Irvine).

\section{Competing interests}

$\mathrm{EH}$ and PSE are employees of Population Bio. BRC, SW, RK, ST, and EC are employees of Ambry Genetics. The other authors declare no competing interests.

\section{Patient consent for publication}

Parental/guardian consent obtained. 


\section{Ethics approval}

Ethics approval for this study was obtained from the University of California, Irvine.

\section{Provenance and peer review}

Not commissioned; externally peer-reviewed 


\section{REFERENCES}

1. Wirth C, Brandt U, Hunte C, Zickermann V. Structure and function of mitochondrial complex I. Biochim Biophys Acta. 2016;1857(7):902-14.

2. Thompson K, Collier JJ, Glasgow RIC, Robertson FM, Pyle A, Blakely EL, Alston $\mathrm{CL}$, Olahova M, McFarland R, Taylor RW. Recent advances in understanding the molecular genetic basis of mitochondrial disease. J Inherit Metab Dis. 2019.

3. Calvo SE, Tucker EJ, Compton AG, Kirby DM, Crawford G, Burtt NP, Rivas M, Guiducci C, Bruno DL, Goldberger OA, Redman MC, Wiltshire E, Wilson CJ, Altshuler D, Gabriel SB, Daly MJ, Thorburn DR, Mootha VK. High-throughput, pooled sequencing identifies mutations in NUBPL and FOXRED1 in human complex I deficiency. Nat Genet. 2010;42(10):851-8.

4. Formosa LE, Dibley MG, Stroud DA, Ryan MT. Building a complex complex: Assembly of mitochondrial respiratory chain complex I. Semin Cell Dev Biol. 2018;76:154-62.

5. Ghezzi D, Zeviani M. Human diseases associated with defects in assembly of OXPHOS complexes. Essays Biochem. 2018;62(3):271-86.

6. Bych K, Kerscher S, Netz DJ, Pierik AJ, Zwicker K, Huynen MA, Lill R, Brandt U, Balk J. The iron-sulphur protein Ind1 is required for effective complex I assembly. EMBO J. 2008;27(12):1736-46.

7. Sheftel AD, Stehling O, Pierik AJ, Netz DJ, Kerscher S, Elsässer HP, Wittig I, Balk J, Brandt U, Lill R. Human ind1, an iron-sulfur cluster assembly factor for respiratory complex I. Mol Cell Biol. 2009;22:6059-73.

8. Tucker EJ, Mimaki M, Compton AG, McKenzie M, Ryan MT, Thorburn DR. Nextgeneration sequencing in molecular diagnosis: NUBPL mutations highlight the challenges of variant detection and interpretation. Hum Mutat. 2012;33(2):411-8.

9. Karczewski K, Francioli L, Tiao G, Cummings BB, Alföldi J, Wang Q, Collins RL, Laricchia KM, Ganna A, Birnbaum DP, Gauthier LD, Brand H, Solomonson M, Watts NA, Rhodes D, Singer-Berk M, England EM, Seaby EG, Kosmicki JA, Walters RK, Tashman K, Farjoun Y, Banks E, Poterba T, Wang A, Seed C, Whiffin N, Chong JX, Samocha KE, Pierce-Hoffman E, Zappala Z, O'Donnell-Luria AH, Vallabh Minikel E, Weisburd B, Lek M, Ware JS, Vittal C, Armean IM, Bergelson L, Cibulskis K, Connolly KM, Covarrubias M, Donnelly S, Ferriera S, Gabriel S, Gentry J, Gupta N, Jeandet T, Kaplan D, Llanwarne C, Munshi R, Novod S, Petrillo N, Roazen D, Ruano-Rubio V, Saltzman A, Schleicher M, Soto J, Tibbetts K, Tolonen C, Wade G, Talkowski ME, The Genome Aggregation Database Consortium, Neale BM, Daly MJ, MacArthur DG Variation across 141,456 human exomes and genomes reveals the spectrum of loss-offunction intolerance across human protein-coding genes bioRxiv preprint first posted online Jan 28, 2019; doi: https://www.biorxiv.org/content/10.1101/531210v3. 2019. 10. Kevelam SH, Rodenburg RJ, Wolf NI, Ferreira P, Lunsing RJ, Nijtmans LG, Mitchell A, Arroyo HA, Rating D, Vanderver A, van Berkel CG, Abbink TE, Heutink P, van der Knaap MS. NUBPL mutations in patients with complex I deficiency and a distinct MRI pattern. Neurology. 2013;80(17):1577-83. 
11. Balint B, Charlesworth G, Stamelou M, Carr L, Mencacci NE, Wood NW, Bhatia KP. Mitochondrial complex I NUBPL mutations cause combined dystonia with bilateral striatal necrosis and cerebellar atrophy. Eur J Neurol. 2019;26(9):1240-3.

12. Maclean AE, Kimonis VE, Balk J. Pathogenic mutations in NUBPL affect complex I activity and cold tolerance in the yeast model Yarrowia lipolytica. Hum Mol Genet. 2018;27(21):3697-709.

13. Protasoni M, Bruno C, Donati MA, Mohamoud K, Severino M, Allegri A, Robinson AJ, Reyes A, Zeviani M, Garone C. Novel compound heterozygous pathogenic variants in nucleotide-binding protein like protein (NUBPL) cause leukoencephalopathy with multi-systemic involvement. Mol Genet Metab. 2020;129(1):26-34.

14. Tenisch EV, Lebre AS, Grevent D, de Lonlay P, Rio M, Zilbovicius M, Funalot B, Desguerre I, Brunelle F, Rotig A, Munnich A, Boddaert N. Massive and exclusive pontocerebellar damage in mitochondrial disease and NUBPL mutations. Neurology. 2012;79(4):391.

15. Friederich MW, Perez FA, Knight KM, Van Hove RA, Yang SP, Saneto RP, Van Hove JLK. Pathogenic variants in NUBPL result in failure to assemble the matrix arm of complex I and cause a complex leukoencephalopathy with thalamic involvement. $\mathrm{Mol}$ Genet Metab. 2020;129(3):236-42.

16. Farwell KD, Shahmirzadi L, El-Khechen D, Powis Z, Chao EC, Tippin Davis B, Baxter RM, Zeng W, Mroske C, Parra MC, Gandomi SK, Lu I, Li X, Lu H, Lu HM, Salvador D, Ruble D, Lao M, Fischbach S, Wen J, Lee S, Elliott A, Dunlop CL, Tang S. Enhanced utility of family-centered diagnostic exome sequencing with inheritance model-based analysis: results from 500 unselected families with undiagnosed genetic conditions. Genet Med. 2015;17(7):578-86.

17. Posey JE, Rosenfeld JA, James RA, Bainbridge M, Niu Z, Wang X, Dhar S, Wiszniewski W, Akdemir ZH, Gambin T, Xia F, Person RE, Walkiewicz M, Shaw CA, Sutton VR, Beaudet AL, Muzny D, Eng CM, Yang Y, Gibbs RA, Lupski JR, Boerwinkle E, Plon SE. Molecular diagnostic experience of whole-exome sequencing in adult patients. Genet Med. 2016;18(7):678-85.

18. Farber-Katz S, Hsuan V, Wu S, Landrith T, Vuong H, Xu D, Li B, Hoo J, Lam S, Nashed S, Toppmeyer D, Gray P, Haynes G, Lu HM, Elliott A, Tippin Davis B, Karam R. Quantitative Analysis of BRCA1 and BRCA2 Germline Splicing Variants Using a Novel RNA-Massively Parallel Sequencing Assay. Front Oncol. 2018;8:286.

19. Jarrett SG, Rohrer B, Perron NR, Beeson C, Boulton ME. Assessment of mitochondrial damage in retinal cells and tissues using quantitative polymerase chain reaction for mitochondrial DNA damage and extracellular flux assay for mitochondrial respiration activity. Methods Mol Biol. 2013;935:227-43.

20. Wydro MM, Balk J. Insights into the pathogenic character of a common NUBPL branch-site mutation associated with mitochondrial disease and complex I deficiency using a yeast model. Dis Model Mech. 2013;6(5):1279-84.

21. Girirajan S, Rosenfeld JA, Cooper GM, Antonacci F, Siswara P, Itsara A, Vives L, Walsh T, McCarthy SE, Baker C, Mefford HC, Kidd JM, Browning SR, Browning BL, Dickel DE, Levy DL, Ballif BC, Platky K, Farber DM, Gowans GC, Wetherbee JJ, Asamoah A, Weaver DD, Mark PR, Dickerson J, Garg BP, Ellingwood SA, Smith R, Banks VC, Smith W, McDonald MT, Hoo JJ, French BN, Hudson C, Johnson JP, Ozmore JR, Moeschler JB, Surti U, Escobar LF, El-Khechen D, Gorski JL, Kussmann J, 
Salbert B, Lacassie Y, Biser A, McDonald-McGinn DM, Zackai EH, Deardorff MA, Shaikh TH, Haan E, Friend KL, Fichera M, Romano C, Gecz J, DeLisi LE, Sebat J, King MC, Shaffer LG, Eichler EE. A recurrent 16p12.1 microdeletion supports a two-hit model for severe developmental delay. Nat Genet. 2010;42(3):203-9.

22. Parikh S, Karaa A, Goldstein A, Bertini ES, Chinnery PF, Christodoulou J, Cohen $\mathrm{BH}$, Davis RL, Falk MJ, Fratter C, Horvath R, Koenig MK, Mancuso M, McCormack S, McCormick EM, McFarland R, Nesbitt V, Schiff M, Steele H, Stockler S, Sue C, Tarnopolsky M, Thorburn DR, Vockley J, Rahman S. Diagnosis of 'possible' mitochondrial disease: an existential crisis. J Med Genet. 2019;56(3):123-30.

23. Baide-Mairena H, Gaudo P, Marti-Sanchez L, Emperador S, Sanchez-Montanez A, Alonso-Luengo O, Correa M, Grau AM, Ortigoza-Escobar JD, Artuch R, Vazquez E, Del Toro M, Garrido-Perez N, Ruiz-Pesini E, Montoya J, Bayona-Bafaluy MP, PerezDuenas B. Mutations in the mitochondrial complex I assembly factor NDUFAF6 cause isolated bilateral striatal necrosis and progressive dystonia in childhood. Mol Genet Metab. 2019;126(3):250-8.

24. Simon MT, Eftekharian SS, Stover AE, Osborne AF, Braffman BH, Chang RC, Wang RY, Steenari MR, Tang S, Hwu PW, Taft RJ, Benke PJ, Abdenur JE. Novel mutations in the mitochondrial complex I assembly gene NDUFAF5 reveal heterogeneous phenotypes. Mol Genet Metab. 2019;126(1):53-63.

25. Alston CL, Veling MT, Heidler J, Taylor LS, Alaimo JT, Sung AY, He L, Hopton S, Broomfield A, Pavaine J, Diaz J, Leon E, Wolf P, McFarland R, Prokisch H, Wortmann SB, Bonnen PE, Wittig I, Pagliarini DJ, Taylor RW. Pathogenic Bi-Allelic Mutations in NDUFAF8 Cause Leigh Syndrome with an Isolated Complex I Deficiency. Am J Hum Genet. 2019.

26. Enns GM, Cohen BH. Clinical Trials in Mitochondrial Disease: An Update on EPI743 and RP103. Journal of Inborn Errors of Metabolism and Screening 2017;5:1-7. 27. Enns GM, Kinsman SL, Perlman SL, Spicer KM, Abdenur JE, Cohen BH, Amagata A, Barnes A, Kheifets V, Shrader WD, Thoolen M, Blankenberg F, Miller G. Initial experience in the treatment of inherited mitochondrial disease with EPI-743. Mol Genet Metab. 2012;105(1):91-102. 


\section{FIGURE LEGENDS}

Figure 1 New patients with NUBPL disease. (A) Pedigree charts for five new patients (four families). Circles indicate females and squares males, arrow $\mathrm{P}=$ Proband. Family 4 has a suspected patient (see text). (B) Cross section of brain MRIs in all subjects (Patients 1A and 1B show MRIs for two different ages). Cerebellar and pons hypoplasia are noted (and cerebellar atrophy) except in Patient 4, who had normal brain anatomy except slightly prominent Sylvian fissures/subarchnoid spaces. (C) Cerebellar brain biopsy for Patient $1 \mathrm{~A}$ at age one year with hematoxylin and eosin (H\&E) stain (left panel) and neurofilament (NF) antibody stain (right panel, same region as left panel). The biopsy shows abnormal foliar architecture with large and irregularly distributed Purkinje neurons (highlighted with Neurofilament protein immunohistochemistry), Bergmann gliosis, and widespread karyorrhexis of the internal granular cell layer.

Figure 2 Alternatively spliced transcripts levels are higher in NUBPL c.815-27T >C carriers. (A) CloneSeq results for the splice variants observed in Family 1 are displayed as Sashimi plots, wherein transcript levels are quantified as Reads Per Kilobase of transcript, per Million mapped reads (RPKM). These provide an absolute number of aligned reads (numbers are indicated for each family member) that enables comparison of exon usage across the carriers (Sister+, Proband+, Father+), non-carriers (Mother-, Sister-), and controls (Blood control-, Brain control-). In addition to normal transcript, two alternate transcripts were observed: r.815_897del83 (exon 10 skipping) and r.81572_815-1ins72 (partial inclusion of intron 9). (B) The relative expression of the two alternatively spliced transcripts are displayed as Percent Splicing Index (PSI). Higher levels of both alternate transcripts were observed only in c.815-27T $>C$ carriers.

Figure 3 Mitochondrial respiration is impaired in NUBPL patient fibroblasts. (A) Oxygen consumption rate (OCR) traces were measured in fibroblast cell lines from NUBPL patients $1 \mathrm{~A}, 1 \mathrm{~B}$, and 2 and a control cell line using the Seahorse XF Cell Mito Stress Test. Three measurements were taken for each stage of the assay: the initial OCR; 
OCR after injection of oligomycin; OCR after injection of FCCP; and OCR after injection of rotenone + antimycin A. The OCR values are normalized by total protein for each well. (B) Basal respiration and spare respiratory capacity for NUBPL patient and control fibroblast cell lines, calculated using Mito Stress Test report generator software. Basal respiration is calculated by subtracting the non-mitochondrial OCR (lowest measurement after the injection of rotenone + antimycin A) from the initial OCR (last measurement before the injection of oligomycin). Spare respiratory capacity is calculated by subtracting the initial OCR from the maximal OCR (maximal measurement after injection of FCCP). Error bars are the standard deviation; statistical significance was calculated using student's t-test. The results show that the spare respiratory capacity for all three patients was less than control.

Figure 4 p.V182A substitution does not impact function of the NUBPL homolog in a yeast model. (A) Alignment of the amino acid sequence surrounding valine 182 (V182) in NUBPL from human (NP_079428.2) and the homologous Ind1 protein from Yarrowia lipolytica (XP_501064.1). Conserved residues are shaded black. Position V182 is semiconserved (shaded grey), with a methionine present at the corresponding position in Ind1 (M180). (B) Complex I levels in the Ind1 M180V and M180A variants compared to control (ind1 deletion mutant expressing the wild-type IND1 gene from a plasmid and an ind 1 deletion mutant ind $1 \Delta$ ). Complex I was visualised by NADH/NBT staining of BlueNative Poly-Acrylamide Gel Electrophoresis (BN-PAGE) gels. Ind1 and Aco1 protein levels were detected by SDS-PAGE and immunoblot analysis. (C) Assembly intermediates of complex I containing the NUCM subunit (NDUFS2 in human), visualised by BN-PAGE and immunoblotting. (D) Growth of the indicated Yarrowia yeast strains at normal and cold temperatures. Images show a serial dilution of cultures that were spotted onto agar plates. 